\title{
VULNERABILIDAD A LA CONTAMINACIÓN DE LA MICRO-CUENCA DEL RÍO TRINTARA WASPAM RÍO COCO
}

Bency Taylor Samuel ${ }^{[1]}$

Francis Martínez Manuel ${ }^{[2]}$ Enrique Cordón Suárez ${ }^{[3]}$

\section{Resumen}

Esta investigación presenta los resultados en la micro-cuenca del río Trintara, perteneciente a la cuenca del río Coco, en el Municipio de Waspam, RAAN, Nicaragua. El objetivo fue analizar la vulnerabilidad a la contaminación del recurso hídrico y proponer alternativas de manejo adecuado para reducir la contaminación. En la metodología documental se han descrito las características socioeconómicas y biofísicas, así como la contaminación e informes de trabajo del municipio. Para el análisis de la vulnerabilidad global a la contaminación de la micro cuenca se tomó como base la metodología descrita por Watler (2008), que presenta la vulnerabilidad, basada en los criterios de Wilches-Chaux (1993).

Los resultados muestran que la vulnerabilidad global a la contaminación de la micro-cuenca del río Trintara es de 2.98 (61.78 \%), caracterizándose con una alta vulnerabilidad a la contaminación. La vulnerabilidad social, educativa, política-institucional y económica, están en el rango de alta vulnerabilidad con un valor superior a tres, todas tienen que ver con acciones directas de la población. En cambio, la vulnerabilidad física y ecológica presenta una valoración de vulnerabilidad media, con un valor inferior a tres. Se recomienda la aplicación de medidas de ordenación de la micro cuenca de manera institucionalizada con el acompañamiento de las comunidades.

Palabras clave: Vulnerabilidad, alternativas, manejo, contaminación.

\section{Summary}

This research presents the results in the micro-basin of Trintara River, which is located in the Coco River Basin, Municipality of Waspam, RAAN, Nicaragua. The purpose was to analyze the vulnerability to water resources pollution and propose alternative management to reduce such contamination. The methodology describes the socioeconomic and biophysical characteristics, also the reports of the municipality. For the analysis of global vulnerability to pollution of the micro-basin, we used the

[1] Ing. Agroforestal, autor principal del artículo. miskut@yahoo.com

[2] Ing. Agroforestal, autor principal del artículo. mafr@yahoo.com

[3] PhD. Docente investigador Tutor del estudio. encordon@yahoo.com 
methodology described by Watler (2008), which presents the vulnerability based on Wilches-Chaux criteria's (1993).

The results show that the global vulnerability to pollution of the micro-basin of Trintara River is 2.98 (61.78\%), which is characterized as high. Social, educational, political-institutional and economic vulnerability are in the range of high with a rate above three, all of which are related to direct actions of the population. Instead, the physical and ecological vulnerability presents a moderate vulnerability assessment, with a rate less than three. The application of institutionalize management measures is recommended in the micro-basin with community accompaniment.

Keywords: Vulnerability, alternatives, management, pollution.

\section{Introducción}

El río Trintara posee una extensión aproximada de 4,646.23 ha, cubriendo una franja de bosque de pinares. El $18 \%$ de esta área es habitada por personas de los barrios Pancasán, 4 de Mayo y 1ro. de Mayo del sector urbano, también sirve de lindero con la comunidad vecina de ulwas. Históricamente se ha utilizado por la población para consumo de agua, pesca, extracción de materiales selectos, extracción de maderas, las prácticas agrícolas, entre otros.

Con el aumento demográfico y la falta de políticas municipales de ordenación urbana, la micro cuenca ha estado sufriendo los impactos directos de la población que la habita. Esto conlleva a un mayor deterioro de la fuente hídrica, mayor sedimentación, deterioro de los ecosistemas y calidad del agua, por tanto hay mayor vulnerabilidad a la contaminación de las aguas superficiales. Se espera que los resultados de este estudio sea la base para la planificación, afín de conservar y proteger esta fuente de agua en la ciudad de Waspam. Tal razón es motivo de la elección del tema de trabajo monográfico.

\section{Revisión de literatura}

\section{Cuencas hidrográficas e hidrológicas}

Según Ramakrishna (1997) citado por Watler (2008:10), una cuenca hidrográfica es un área natural donde el agua proveniente de la precipitación forma un curso principal de agua.

Es la unidad fisiográfica conformada por el conjunto de los sistemas de cursos de agua definidos por el relieve. Los límites de la cuenca o divisoria de aguas se definen naturalmente y corresponden a las partes más altas del área que encierra un río. 
La definición de cuenca hidrológica es más integral que la de cuenca hidrográfica. Ya que son unidades morfológicas integrales, además de incluir todo el concepto de cuencas hidrográfica, abarcan en su contenido toda la estructura hidrológica subterránea del acuífero como un todo (Faustino 2007) citado por Watler (2008:11).

\section{La contaminación de las cuencas como un problema ambiental}

Según Lozano (1998:76), la problemática de contaminación de las cuencas es una cuestión ambiental en la cual se expresa una percepción históricamente reciente, referida a la concentración exagerada de riqueza y la manifestación de la pobreza.

\section{La cuenca en el marco legal}

En el artículo 96 de la ley de aguas nacionales No. 620, asegura que es de interés social asegurar la calidad de los cuerpos de aguas nacionales, a través de la promoción y ejecución de las medidas y acciones necesarias para su debida y permanente protección y conservación. Se prohíbe la tala o corte de árboles o plantas de cualquier especie, que se encuentren dentro de un área de 200 metros a partir de las riberas de los ríos y costas de los lagos y lagunas a fin de proteger el recurso hídrico existente.

De la misma manera en el artículo 24 de la Ley No. 559, Ley contra el delito ambiental, establece que las personas que pesque con elementos explosivos, venenos $\mathrm{u}$ otra forma de pesca destructiva serán sancionadas de dos a cuatro años de prisión y el decomiso de instrumentos y equipos utilizados.

\section{Caracterización biofísica y socioeconómica}

Cuadro No. 1. Clasificación de cuencas en función del orden de drenaje y área que las encierran

\begin{tabular}{l|l|l|}
\hline \multicolumn{1}{|c|}{ Unidad } & \multicolumn{1}{c|}{ No. de orden } & \multicolumn{1}{c|}{ Área (Km2) } \\
\hline Micro-Cuenca & 1,2 y 3 & $10-100$ \\
\hline Sub-Cuenca & 4 y 5 & $100-700$ \\
\hline Cuenca & 6,70 más & Mayor de 700 \\
\hline
\end{tabular}

\section{Clasificación de cuencas por su sistema de drenaje}

Las cuencas hidrográficas pueden clasificarse en:

- Arreicas: Cuando no logran drenar a un río, mar o lago, sus aguas se pierden por evaporación o infiltración sin llegar a formar escurrimiento subterráneo.

- Criptorreicas: Cuando sus redes de drenaje superficial no tienen un sistema organizado o aparente y corren como ríos subterráneos. 
- Endorreicas: Cuando sus aguas drenan a un embalse o lago, sin llegar al mar.

- Exorreicas: Cuando las vertientes conducen las aguas a un sistema mayor de drenaje como un gran río o mar.

\section{Vulnerabilidad y riesgo a la contaminación de aguas}

\section{Vulnerabilidad}

La OPS (1987) citado por (Watler 2008:8), la define como la medida de la debilidad de un componente para resistir el impacto de las amenazas. Únicamente para efectos de estudios se divide la vulnerabilidad global en distintas vulnerabilidades. Sin embargo, es indispensable señalar que cada una de ellas constituye apenas un ángulo particular para analizar el fenómeno global y las diferencias, debido a que están estrechamente interconectadas entre sí. Es decir, difícilmente podríamos entender, por ejemplo, la vulnerabilidad física sin considerar una función de la vulnerabilidad económica y de la política, o esta última sin tomar en cuenta la vulnerabilidad social, ecológica, etc. (Wilches-Chaux, 1993).

\section{Tipos de vulnerabilidad}

Según Wilches-Chaux (1989), sostiene que una sociedad puede enfrentar distintas vulnerabilidades y las clasifica de la siguiente manera:

- Vulnerabilidad natural: Se refiere a los ecosistemas de los distintos países se incrementó diferencialmente, provocando la resistencia de la población a condiciones ambientales severas y a veces haciéndola más vulnerable frente a ellas.

- Vulnerabilidad física: Se refiere a la localización de la población en zona de riesgo físico, condición provocada por la pobreza y la falta de oportunidades para una ubicación de menor riesgo (condiciones ambientales y de los ecosistemas, localización de asentamientos humanos en zonas de riesgo).

- Vulnerabilidad económica: Se observa una relación indirecta entre los ingresos en los niveles nacional, regional, local o poblacional y el impacto de los fenómenos físicos extremos. Es decir, la pobreza aumenta el riesgo de desastre (vulnerabilidad de los sectores más deprimidos, desempleo, insuficiencia de ingresos, explotación, inestabilidad laboral, dificultad de acceso a los servicios de educación, salud).

- Vulnerabilidad social: Se produce un grado deficiente de organización y cohesión interna de la sociedad bajo riesgo, que limita su capacidad de prevenir, mitigar o responder a situaciones de desastres (tipo de acceso al saneamiento 
ambiental, nutrición infantil, servicios básicos, que permitan la recuperación de los daños ocurridos).

- Vulnerabilidad política: Concentración de la toma de decisiones, centralismo en la organización gubernamental y la debilidad en la autonomía de los ámbitos regionales, locales y comunitarios, lo que impide afrontar los problemas. (Autonomía en el poder de decisión y de solucionar problemas).

- Vulnerabilidad técnica: Se refiere a las inadecuadas técnicas de construcción de edificios e infraestructura básica utilizadas en áreas de riesgo (incapacidad de control y manejo de las tecnologías frente a los riesgos).

- Vulnerabilidad ideológica: Alude a la forma y concepción del mundo y el medio ambiente donde se habita y con el cual se relaciona y la posibilidad de enfrentar los problemas. La pasividad, fatalismo, presencia de mitos, aumentan la vulnerabilidad de la población.

- Vulnerabilidad educativa: Falta de programas educativos que proporcionen información sobre el medio ambiente, el entorno, los desequilibrios y las formas adecuadas de comportamiento individual o colectivo en caso de amenaza o de situación de desastre (conocimiento de las realidades locales y regionales para hacer frente a los problemas).

- Vulnerabilidad cultural: Se refiere a la forma en que los individuos y la sociedad conforman el conjunto nacional y el papel que juegan los medios de comunicación en la consolidación de estereotipos o en la transmisión de información relacionada con el medio ambiente y los potenciales o reales desastres (influencia de la personalidad de los habitantes que se identifican con un modelo de sociedad, influencias por los medios masivos de comunicación frente a los riesgos).

- Vulnerabilidad ecológica: Relacionada a la convivencia con el medio ambiente, sin la dominación por destrucción. Vulnerabilidad de los ecosistemas frente a los efectos directos o indirectos de la acción humana, y por otra, altos riesgos para las comunidades que los explotan o habitan.

- Vulnerabilidad institucional: La obsolescencia y rigidez de las instituciones, en las cuales la burocracia, la prevalencia de la decisión política, el dominio de criterios personalistas, impiden respuestas adecuadas y ágiles a la realidad existente y demoran el tratamiento de los riesgos o sus efectos.

\section{Tipos de contaminación}

Según Guerra (2004), clasifica la contaminación en cuatro tipos:

La contaminación natural: Consiste en la presencia de determinadas sustancias en el agua sin que intervenga la acción humana. 
La contaminación de origen urbano: Es el resultado del uso del agua en viviendas, actividades comerciales y de servicios, lo que genera aguas residuales, que son devueltas al receptor con un contenido de residuos fecales, deshechos de alimentos y en la actualidad con un incremento de productos químicos.

La contaminación de origen agrícola: Deriva del uso de plaguicidas, pesticidas, biocidas, fertilizantes y abonos, que son arrastrados por el agua de riego, llevando consigo sales compuestas de nitrógeno, fósforo, azufre y trazas de elementos de órganos clorados que pueden llegar al suelo por lixiviado y contaminar las aguas subterráneas.

La contaminación de origen industrial: Es una de las que produce un mayor impacto, por la gran variedad de materiales y fuentes contaminantes al agua.

\section{Manejo y gestión de cuenca}

Según Cosgrove, citado por Burton, (2003: 5), el concepto de Gestión Integral de Recursos Hídricos (GIRH), en contraste con el concepto tradicional fragmentado de manejo de agua (el cual tiene sólo énfasis para satisfacer su demanda), incorpora dos dimensiones fundamentales: i) el sistema natural, el cual representa una componente de importancia vital para la disponibilidad de la cantidad y calidad del recurso así como de una amplia gama de servicios ambientales que provee; ii) la dimensión humana la cual fundamentalmente determina el uso del recurso, la contaminación y degradación del recurso y, determina cuales deben ser las prioridades de desarrollo.

\section{Materiales y métodos}

\section{Tipo de estudio}

El estudio es descriptivo y de corte transversal, el área comprende la ciudad de Waspam y abarca 3 barrios afectados y una comunidad vecina (ulwas).

\section{Universo y muestra}

El universo del estudio comprende toda el área de la micro-cuenca Trintara Tigni que cuenta con un área aproximado de 4,646.23 ha y una longitud de 10,637.18 m. La muestra se obtuvo representativamente de cada ángulo de vulnerabilidad de acuerdo a sus variables e indicadores en estudio. 


\section{Caracterización socioeconómica y biofísica}

Se recopiló información socioeconómica sobre: población por sexo, organización comunitaria, actividades económicas, servicios básicos existentes: salud, educación (nivel de instrucción, analfabetismo, infraestructuras educativas), transporte, infraestructuras y viviendas. La información biofísica incluyó información climática: temperatura, precipitación, vientos y humedad relativa; información geomorfológica: relieve, tipo de suelos, pendientes, entre otros; y la información bióptica: fauna y flora, uso del suelo, e información hidrográfica o hidrológica. Para ambos, las fuentes de informaciones fueron: diagnósticos socioeconómicos del municipio, monografía, informes. Las técnicas fueron entrevistas y encuestas.

\section{Vulnerabilidad global}

Se constituyó bajo la metodología descrita en la escuela de Postgrado del Centro Agronómico Tropical de Investigación y Enseñanza (CATIE, citado por Watler, 2008), para presentar la vulnerabilidad global, basada en los conceptos, criterios y propuesta de Wilches-Chaux (1989,1993).

Cuadro No. 2. Tipos de vulnerabilidad

\begin{tabular}{|l|l|l|}
\hline No. & Tipo de vulnerabilidad & \multicolumn{1}{c|}{ Variables } \\
\hline 1 & Física & $\begin{array}{l}\text { Asentamiento, sistema séptico, basureros, comercios, gaso- } \\
\text { lineras, extracción de materiales selectos, aguas residuales } \\
\text { y tren de aseo. }\end{array}$ \\
\hline 2 & Política-Institucional & $\begin{array}{l}\text { Proyectos y programas, participación comunitaria, lideraz- } \\
\text { go y leyes. }\end{array}$ \\
\hline 3 & Ecológica & $\begin{array}{l}\text { Agroquímicos, erosión, tipos de suelos, deforestación, } \\
\text { agricultura, ganadería, biofísicos, contaminación, áreas de } \\
\text { intervención en las nacientes. }\end{array}$ \\
\hline 6 & Económica & $\begin{array}{l}\text { Capacidad económica, fuentes de empleo, y desempleo, } \\
\text { instrumentos económicos. }\end{array}$ \\
\hline 5 & Social & $\begin{array}{l}\text { Instituciones, participación de productores, apoyo socie- } \\
\text { dad civil, salud, servicios públicos. }\end{array}$ \\
\hline
\end{tabular}


Cuadro No. 3. Caracterización y valoración de la vulnerabilidad

\begin{tabular}{|c|c|}
\hline Caracterización de la vulnerabilidad & Valoración de la vulnerabilidad \\
\hline Muy alta & 4 \\
\hline Alta & 3 \\
\hline Media & 2 \\
\hline Baja & 1 \\
\hline Muy baja & 0 \\
\hline NA (No Aplica) & NA \\
\hline
\end{tabular}

Cuadro No. 4. Escala de valoración de la vulnerabilidad

\begin{tabular}{|c|c|}
\hline Escala porcentual de la vulnerabilidad & $\begin{array}{c}\text { Caracterización de la } \\
\text { vulnerabilidad }\end{array}$ \\
\hline $0,00-19,99$ & Muy baja \\
\hline $20,00-39,99$ & Baja \\
\hline $40,00-59,99$ & Media \\
\hline $60,00-79,99$ & Alta \\
\hline $80,00-100$ & Muy alta \\
\hline
\end{tabular}

Luego se estimará la vulnerabilidad global a la contaminación utilizando la siguiente ecuación: $\boldsymbol{V G}=\sum \mathrm{i} 6=1 \mathrm{xi}^{*} \mathrm{wi}=\left(\mathrm{VF}^{*} \mathrm{o}, 25+\mathrm{VPI}^{*} \mathrm{o,10}+\mathrm{VEC}^{*} \mathrm{0,25}+\mathrm{VEN}^{*} \mathrm{0,15}\right.$ $\left.+\mathrm{VS}^{*} 0,15+\mathrm{VE}^{*} \mathrm{o,10}\right)$

\section{Resultados y discusión}

\section{Aspectos socioeconómicos}

Cuadro No. 5. Datos poblacionales y viviendas

\begin{tabular}{|l|l|r|r|r|r|r|r|}
\hline \multirow{2}{*}{ No. } & \multicolumn{1}{|c|}{ Barrio } & \multirow{2}{*}{ Sector } & \multirow{2}{*}{$\begin{array}{c}\text { No. de } \\
\text { Casas }\end{array}$} & \multirow{2}{*}{$\begin{array}{c}\text { No. de } \\
\text { familias }\end{array}$} & \multicolumn{2}{c|}{$\begin{array}{c}\text { No. de } \\
\text { habitantes }\end{array}$} & \multicolumn{2}{|c|}{ Sexo } \\
\cline { 6 - 9 } & & 1 & 289 & 355 & 1468 & 637 & 831 \\
\hline 1 & 4 de Mayo & 1 & 138 & 153 & 801 & 357 & 444 \\
\hline 2 & Pancasán & & 5 & 8 & 18 & 8 & 10 \\
\hline 3 & $\begin{array}{l}\text { Ulwas 100 m sobre el } \\
\text { río Trintara }\end{array}$ & 0 & 432 & 516 & 2287 & 1002 & 1285 \\
\hline \multicolumn{2}{|c}{ Total } \\
\hline
\end{tabular}

Fuente: Departamento de sanidad e higiene, MINSA 2011. 
Los resultados reflejan que existen un total de 432 viviendas, con 516 familias y 2,287 habitantes en una franja de 100 metros paralelos al río Trintara (información de los tres barrios y comunidad de ulwas). Sin embargo, gran parte de la disposición de las viviendas en el terreno es desordenada y esto implica mayor vulnerabilidad.

Cuadro No. 6. Datos de las infraestructura domiciliar

\begin{tabular}{|c|c|c|c|c|c|c|c|}
\hline \multirow[b]{2}{*}{ No. } & \multirow[b]{2}{*}{ Barrio } & \multicolumn{2}{|c|}{ Pozos } & \multicolumn{2}{|c|}{ Letrinas } & \multicolumn{2}{|c|}{ Tanque séptico } \\
\hline & & 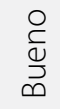 & $\frac{0}{\frac{N}{2}}$ & 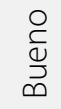 & $\frac{0}{\sum^{\pi}}$ & 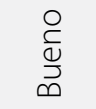 & $\frac{0}{\frac{\pi}{2}}$ \\
\hline 1 & Primero y 4 de Mayo & 141 & 50 & 213 & 29 & 35 & $c$ \\
\hline 2 & Pancasán & 25 & 32 & 68 & 19 & 3 & $\mathrm{c}$ \\
\hline 3 & $\begin{array}{l}\text { Ulwas en la franja de } 100 \\
\text { m sobre el río Trintara }\end{array}$ & 1 & 0 & 4 & 1 & 0 & $c$ \\
\hline & Total & 167 & 82 & 285 & 49 & 38 & $c$ \\
\hline
\end{tabular}

De igual manera, no todas las casas tienen letrinas, por lo que la población hace sus necesidades fisio biológicas al aire libre. Pero también la mayoría de las letrinas existentes están en mal estado o están construidas con materiales inadecuados, muchas de ellas ubicadas entre 10-20 metros en la franja del río. Esto lo convierten al río Trintara Tigni cada vez más vulnerable a la contaminación.

\section{Comercio y actividades económicas}

Cuadro No. 7. Movimiento económico

\begin{tabular}{|l|l|r|r|r|r|}
\hline No. & \multicolumn{1}{|c|}{ Barrio } & Pulpería & Bares & Comedores & Fritanga \\
\hline 1 & 4 de Mayo & 16 & 8 & 3 & 2 \\
\hline 2 & Pancasán & 12 & 1 & 0 & 0 \\
\hline 3 & $\begin{array}{l}\text { Ulwas en la franja de 100 m. } \\
\text { sobre el río Trintara }\end{array}$ & 0 & 0 & 0 & 0 \\
\hline \multicolumn{2}{|c|}{ Total } & 28 & 9 & 3 & 2 \\
\hline
\end{tabular}

Fuente: Alcaldía de Waspam, 2011.

El cuadro refleja que los barrios 4 de Mayo y Pancasán tienen una vida económica dinámica, ya que existe un alto número de pulperías ( 16 y 12 pulperías respectivamente). En segundo plano les siguen los bares, comedores y fritangas. Esto demuestra que existe una vida activa que se deriva del sector informal y que no se encuentran registrados en la municipalidad. Esto hace que las actividades se desarrollen dispersas en la cuenca, provocando mayor daño ambiental. 


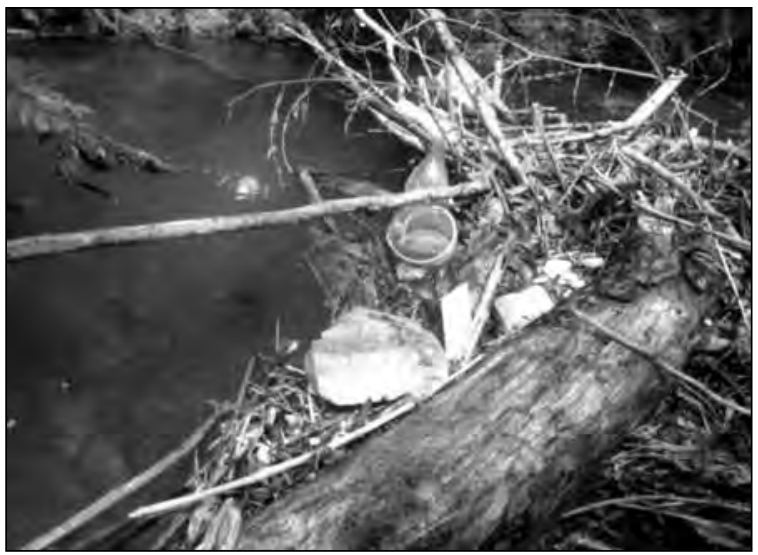

Figura No. 1. Presencia de desechos diversos en el río Trintara.

\section{Servicios básicos}

Salud: Los pobladores de los tres barrios evaluados acuden a los servicios del centro de salud "Oswaldo Padilla"; sin embargo, la atención no cubre la demanda de los tres barrios. Algunos asalariados acuden a la Clínica de las Hermanas de "Santa Inés". Las principales enfermedades son: las gastrointestinales, respiratorias, malaria y el dengue.

Educación: El barrio 4 de Mayo y 1ro. de Mayo cuenta con dos escuelas primarias, una escuela secundaria y una escuela técnica, en cambio el barrio Pancasán cuenta con una sola escuela primaria.

Cuadro No. 8. Datos de educación

\begin{tabular}{|l|r|}
\hline \multicolumn{1}{|c|}{ Población por categoría } & Porcentaje \\
\hline Analfabeto & 36 \\
\hline Finalizada universidad & 1 \\
\hline Finalizada secundaria & 30 \\
\hline Finalizada primaria & 13.1 \\
\hline $\begin{array}{l}\text { Niños menores de 9 años primaria } \\
\text { incompleta }\end{array}$ & 19.9 \\
\hline \multicolumn{1}{|c|}{ Total } & 100 \\
\hline
\end{tabular}

\section{Organización comunitaria}

La organización está representada por un coordinador y un juez (Wihta) y sus suplentes, con la excepción de que sólo un síndico cubre todos los barrios. Sin embargo, sus 
funciones están enfocadas más al área política, no velan por el buen funcionamiento social y ambiental de los barrios, incrementando los niveles de vulnerabilidad.

\section{Aspecto biofísico}

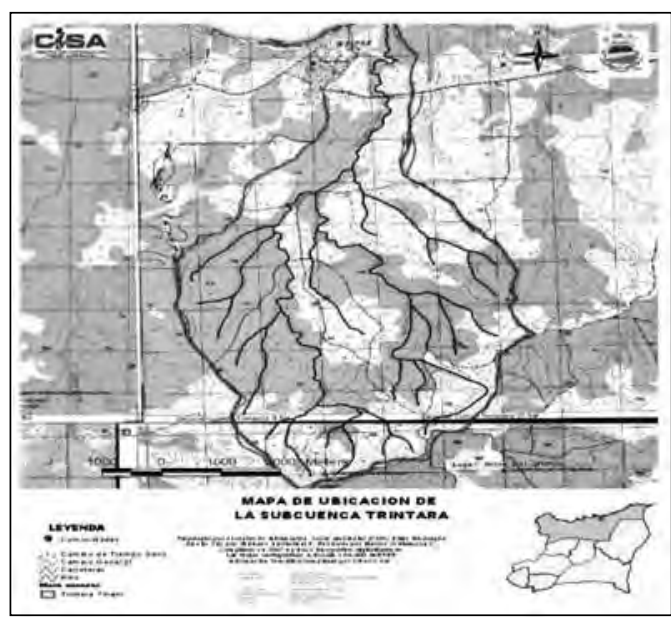

Figura No. 2. Ubicación del área de estudio.

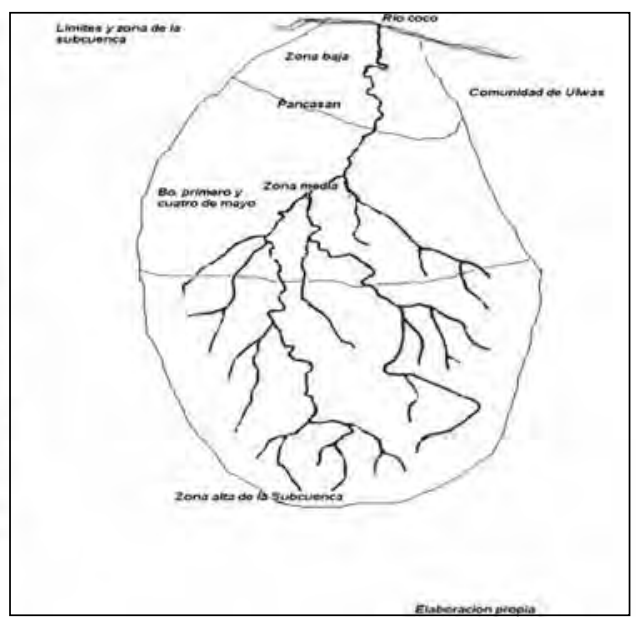

Figura No. 3. División de la cuenca en zonas.

La micro-cuenca del río Trintara se encuentra ubicada en la Región Autónoma del Atlántico Norte (RAAN), cubre 3 barrios de Waspam y que sirve de lindero con la comunidad vecina de los ulwas. Se extiende de Sur a Norte con un área total de 4,646.23 $\mathrm{Ha}$, desemboca sus aguas directamente en el río Coco, en el sector del barrio Santa Inés del segundo embarcadero a 500 metros al Este en el territorio de la comunidad de los ulwas.

- La zona alta se ubica en el empalme Laymus-Tronquera a $10 \mathrm{~km}$ al este, sector del Suroeste de la comunidad de Waspam, la carretera que se dirige hacia Bismuna, aquí se origina la naciente del río Trintara, en la parte alta caracteriza la ausencia de pobladores. Esta zona se caracteriza por la abundante cobertura vegetal con predominante bosque de pinares y latifoliados.

- La zona media cubre entre los barrios 1ro. de Mayo, 4 de Mayo y Pancasán, es un área muy poblada, con abundantes construcciones de madera y concreto, sin embargo muy desordenada.

- La zona baja, abarca desde una parte del barrio Pancasán hasta su desembocadura en el río Coco. Es una zona con una productiva donde los comunitarios de ulwas siembran musáceas, tubérculos, caña, cítricos, cocos y granos básicos de cultivos anuales. 


\section{Fisiografía y relieve}

En común la micro-cuenca del Trintara Tigni se encuentra ubicada en la subprovincia de la Planicie Volcánica Intermedia y Transición a Colina y la Planicie Fluvio Intermedia, con elevaciones divididas en dos partes, la parte baja de o a $50 \mathrm{msnm}$, la parte media y alta con 50 a $100 \mathrm{msnm}$, topografía ligeramente ondulada con pendientes del $5 \mathrm{al}$ $20 \%$. Con precipitación alta, de más de $3000 \mathrm{~mm}$ al año, hay un mayor arrastre de sedimentos y de desechos orgánicos e incrementa la vulnerabilidad.

\section{Clima y precipitación}

En lo común la micro-cuenca Trintara Tigni se encuentra en la Sabana Tropical de pinos, con 9 meses lluviosos, con media anual de precipitación que oscila entre 1,900 y los 3,290 $\mathrm{mm}^{3}$ y estación seca mayor de 2 meses al año.

\section{Uso del suelo}

El suelo es de vocación forestal; sin embargo, algunas familias poseen huertos con cultivos de frutales, musáceas y tubérculos. De las tres zonas la zona media es la más poblada por lo tanto es donde se da el mayor impacto de deterioro y la mayor acumulación de desechos sólidos y líquidos de la cuenca. La zona baja está conformada por bosques latifoliados con característica de bosque de galería y tacotales donde la mayoría de los pobladores de la comunidad de ulwas, tienen establecidos sus parcelas de granos básicos, raíces y tubérculos, musáceas, entre otros. La vulnerabilidad se refleja por el cambio de uso de suelo, erosión y sedimentación en las fuentes hídricas.

\section{Bosque}

Posee abundante bosque que cubre cerca del $45 \%$ del área total (2,090.80 ha). Predomina una vegetación madura a joven de Pinus caribea que posee un diámetro promedio de $30 \mathrm{~cm}$ (DAP) y una altura promedio de $8.8 \mathrm{~m}$ y su densidad sobrepasa de 100 árboles por hectárea. En caso de las especies de hojas anchas (latifoliado) predominan las especies de Manga Larga (Vochysia ferruginea), Santa María (Calophyllum brasiliense), Palo de Agua (Vochysia hondurensis), Níspero (Manilkara achras), Sebo (Virola multiflora) y con mínima presencia de Caoba del Atlántico (Swietenia macrophylla). La vulnerabilidad se refleja por el aprovechamiento ilegal del bosque, sin planes de manejo, prácticas agrícolas dispersas, aumentando así la erosión y mayor arrastre de sedimentos hacia los ríos. 


\section{Hidrología}

Cuadro No. 9. Análisis bacteriológico y físico de agua, micro-cuenca río Trintara

\begin{tabular}{|c|l|l|l|}
\hline Muestra & \multicolumn{1}{|c|}{ Parámetros } & \multicolumn{1}{|c|}{ Resultado } & \multicolumn{1}{c|}{$\begin{array}{c}\text { Valores permisibles por } \\
\text { OMS }\end{array}$} \\
\hline \multirow{4}{*}{ No.1 } & Aspecto & Claro & No se menciona \\
\cline { 2 - 4 } & Turbiedad & 4.2 & 5 UNT \\
\cline { 2 - 5 } & $\mathrm{PH}$ & - & $5.0-8-5$ \\
\cline { 2 - 5 } & Temperatura & - & $18-320 \mathrm{C}$ \\
\cline { 2 - 5 } & $\begin{array}{l}\text { Presencia heces } \\
\text { fecales en \% }\end{array}$ & $\begin{array}{l}100 \% \text {, humano y } \\
\text { animales }\end{array}$ & $\begin{array}{l}\text { o UFC/ml, agua potable, } \\
\text { en Nicaragua 2-4 UFC/ml }\end{array}$ \\
\hline
\end{tabular}

Fuente: MINSA 2011.

\section{Estimación de la vulnerabilidad a la contaminación}

Cuadro No. 10. Resumen de la vulnerabilidad física (VF)

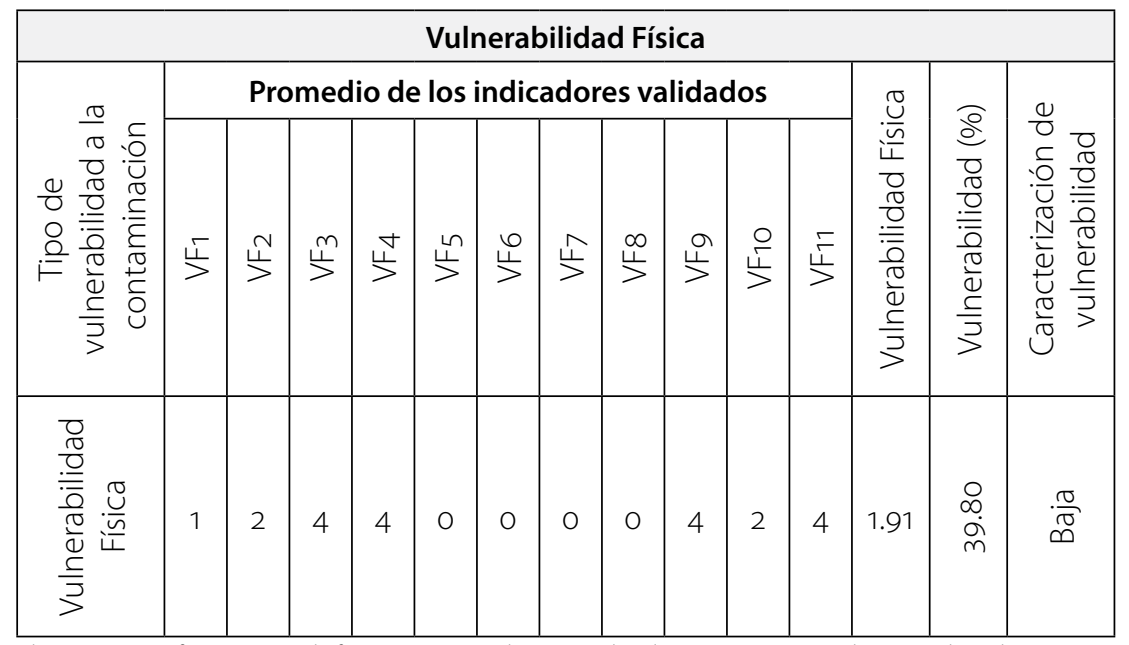

Fuente: Watler, 2008 VF3: Infraestructura de fosa séptica, VF4: basureros ilegales, VF9: extracción de materiales selectas y VF11: tren de aseo con la máxima valoración de vulnerabilidad (4).VF5: vertederos de residuos sólidos o líquidos urbano, VF6: comercio, VF7: pequeñas empresas y VF8: gasolineras con el indicador cero (0.00).

De manera general se observa que la vulnerabilidad física es baja, lo cual es un indicador de que aún no se desarrollan actividades de gran impacto al medio ambiente en la micro-cuenca. La mayoría de las actividades de impacto significativo tienen que ver con las necesidades básicas de las personas y que podrían corregirse con medidas desarrolladas con la municipalidad tales como: letrinas dispersas, extracción de materiales de construcción y basureros dispersos. 
Cuadro No. 11. Resumen de la vulnerabilidad política-institucional

\begin{tabular}{|c|c|c|c|c|c|c|c|c|}
\hline \multicolumn{9}{|c|}{ Vulnerabilidad Política-Institucional } \\
\hline \multirow{2}{*}{$\begin{array}{l}\text { Tipo de vulnerabilidad } \\
\text { a la contaminación }\end{array}$} & \multicolumn{5}{|c|}{$\begin{array}{c}\text { Promedio de los indicadores } \\
\text { validados }\end{array}$} & \multirow{2}{*}{ 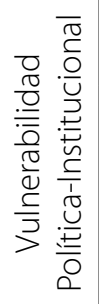 } & \multirow{2}{*}{ 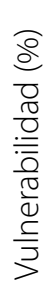 } & \multirow{2}{*}{ 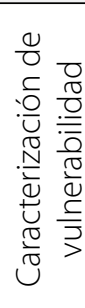 } \\
\hline & VPI-1 & VPI-2 & $\mathrm{VPI}-3$ & $\mathrm{VPI}-4$ & VPI-5 & & & \\
\hline $\begin{array}{c}\text { Vulnerabilidad } \\
\text { Política-Institucional }\end{array}$ & 4 & 4 & 2 & 4 & 2 & 3.20 & $\begin{array}{l}\circ \\
\stackrel{8}{\circ} \\
\stackrel{0}{1}\end{array}$ & $\frac{\pi}{\frac{\pi}{2}}$ \\
\hline
\end{tabular}

Fuente: Watler, 2008 VPI-1: Apoyo de la municipalidad en proyectos y programas de protección, administración y manejo ambiental, VPI-2: Participación de productores y comunitarios, VPI-3: Existencia de liderazgo en la comunidades del área de la subcuenca, VPI-4: Aplicación de la Ley de aguas (No. 620) y Ley contra el delito ambiental (No. 559), VPI-5: Criterios del marco legal.

Se demuestra que hace falta una gestión interinstitucional coordinada y de voluntad por parte de las instituciones competentes en promover programas y proyectos ambientales de protección al recurso agua destinada al consumo humano. Por lo tanto, es urgente desarrollar esfuerzos conjuntos en promover estos programas y proyectos de concientización ambiental, más concretamente dirigido a la protección de las fuentes de aguas y a las áreas de recarga contra la contaminación actual, con acciones que involucre a todos los pobladores de Waspam, no solamente los tres barrios que se encuentran asentadas en el área de la micro- cuenca, sino a la población en general y la comunidad de Ulwas.

Cuadro No. 12. Resumen de la vulnerabilidad ecológica

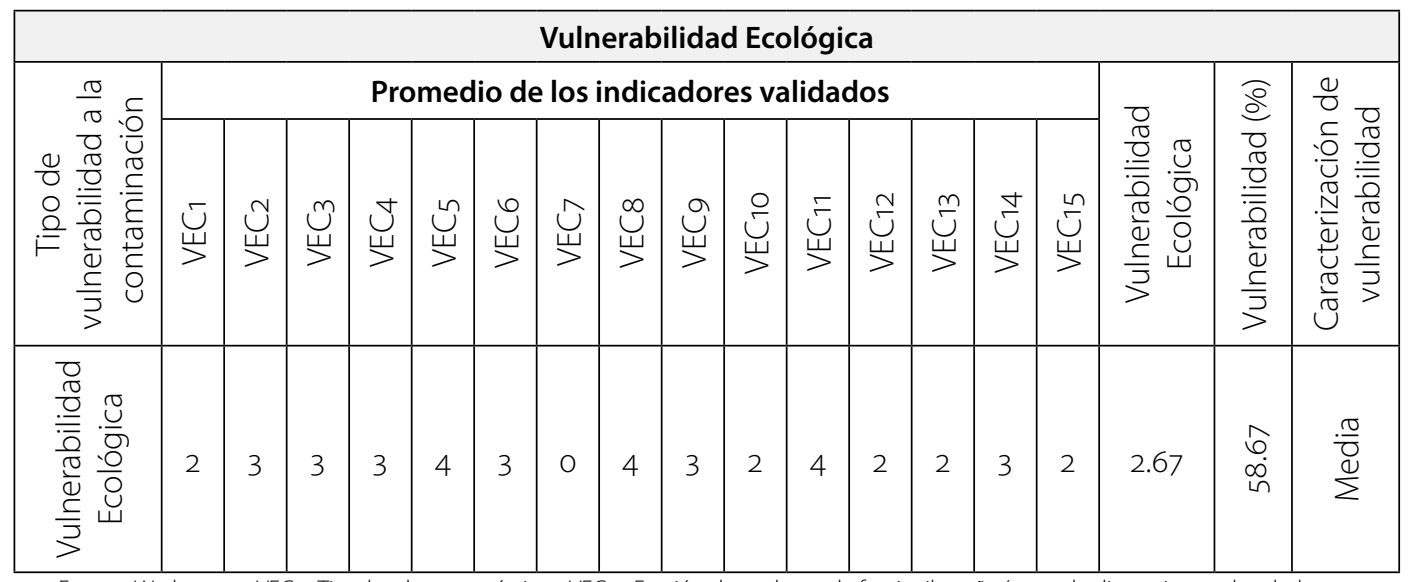

Fuente: Watler, 2008 VEC-1: Tiendas de agroquímicos, VEC-2: Erosión de suelos en la franja ribereña (50 m de distancia a ambos lado al río), VEC-3: Tipo de suelo, VEC-4: Deforestación, VEC-5, 6, 7, 8 y 9: Agricultura, VEC-10 y 11: Ganadería, VEC-12: Biofísico, VEC-13 y 14: Contaminación al río, VEC-15: Áreas de las nacientes. 
Cabe mencionar que cerca del 80\% de la población dentro de la cuenca es desempleada; su sistema de vida y sobrevivencia depende mucho de sus interacción con la naturaleza, en este sentido al no haber un control y regulación del uso de los recursos naturales, así como una política de gestión y de incorporación de la población en el manejo de la cuenca, lo cual tiende a hacer más vulnerable.

Cuadro No. 13. Resumen de la vulnerabilidad económica

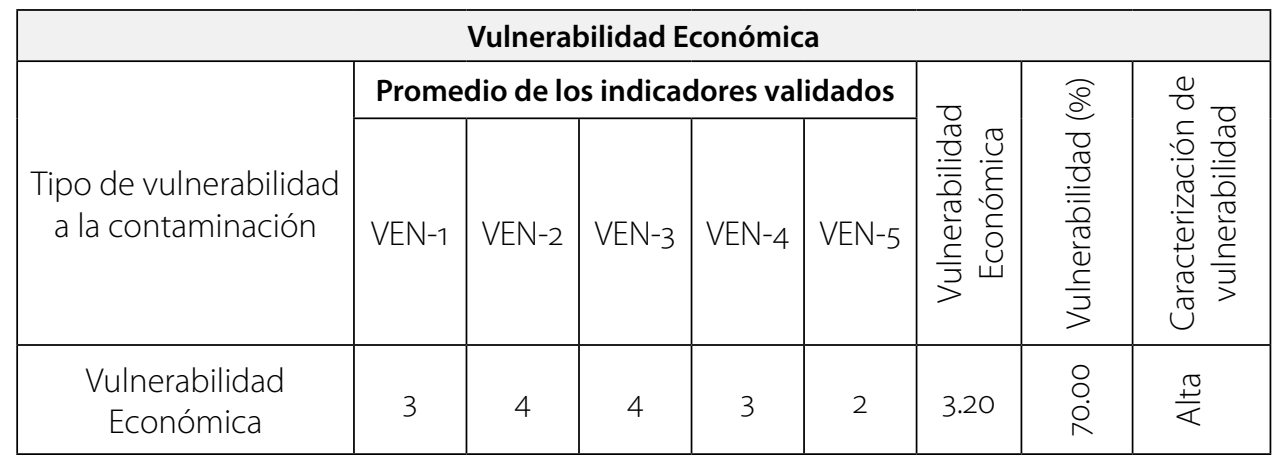

Fuente: Watler, 2008 VEN-1: Áreas de las nacientes, VEN-2: Fuentes de empleo, VEN-3: Instrumentos económicos, VEN-4: Desempleo, VEN-5: Dependencia económica de actividades agropecuaria por familia.

Esta información revela que la poca accesibilidad a los incentivos económicos, más la limitada capacidad económica de los pobladores del barrio 4 de Mayo, Pancasán, 1ro. de Mayo y una parte de la población de la comunidad de ulwas que viven en el área de la micro cuenca, repercute en la expansión de la frontera agrícola-pecuaria y la deforestación ilegal e indiscriminada de las áreas de la micro cuenca, especialmente en la parte alta, donde se encuentran los ojos de agua.

Cuadro No. 14. Resumen de la vulnerabilidad social

\begin{tabular}{|c|c|c|c|c|c|c|c|c|c|c|}
\hline \multicolumn{11}{|c|}{ Vulnerabilidad Social } \\
\hline \multirow[b]{2}{*}{$\begin{array}{l}\text { Tipo de vulnerabilidad } \\
\text { a la contaminación }\end{array}$} & \multicolumn{7}{|c|}{ Promedio de los indicadores validados } & \multirow{2}{*}{ 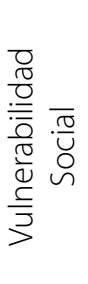 } & \multirow{2}{*}{ 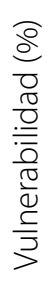 } & \multirow{2}{*}{ 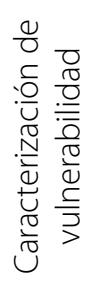 } \\
\hline & VS-1 & VS-2 & VS-3 & VS-4 & VS-5 & VS-6 & VS-7 & & & \\
\hline $\begin{array}{l}\text { Vulnerabilidad } \\
\text { Social }\end{array}$ & 4 & 2 & 4 & 4 & 2 & 4 & 3 & 3.29 & $\stackrel{\stackrel{?}{r}}{\gtrless}$ & $\frac{\mathbb{D}}{\frac{\pi}{4}}$ \\
\hline
\end{tabular}

Fuente: Watler, 2008 VS-1: Instituciones y organizaciones comunales, VS-2: Población de los barrios Pancasán, 4 de Mayo y 1ro. de Mayo, VS-3: Participación de productores, VS-4: Apoyo de la sociedad civil en proyectos comunales de protección, VS-5: Salud, VS-6: Organización social, VS-7: Servicios públicos. 
Esto demuestra que los indicadores sociales valorados desde la óptica de las áreas de protección contra la contaminación de la franja ribereña y/o parte alta de la micro-cuenca, requieren de una mayor atención por parte del gobierno municipal, de las instituciones pertinentes, las organizaciones de la sociedad civil y líderes locales.

Cuadro No. 15. Resumen de la vulnerabilidad educativa

\begin{tabular}{|c|c|c|c|c|c|c|c|}
\hline \multicolumn{8}{|c|}{ Vulnerabilidad Educativa } \\
\hline \multirow{2}{*}{$\begin{array}{c}\text { Tipo de vulnerabilidad } \\
\text { a la contaminación }\end{array}$} & \multicolumn{4}{|c|}{$\begin{array}{c}\text { Promedio de los indicadores } \\
\text { validados }\end{array}$} & \multirow{2}{*}{ 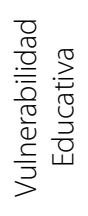 } & \multirow{2}{*}{ 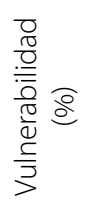 } & \multirow{2}{*}{ 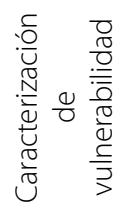 } \\
\hline & VE-1 & $V E-2$ & $V E-3$ & $V E-4$ & & & \\
\hline $\begin{array}{c}\text { Vulnerabilidad } \\
\text { Educativa }\end{array}$ & 4 & 4 & 4 & 2 & 3.50 & $\stackrel{\substack{N \\
\text { H }}}{\wedge}$ & $\stackrel{\stackrel{5}{世}}{<}$ \\
\hline
\end{tabular}

Fuente: Watler, 2008 VE-1: Talleres educativos, VE-2: Educación ambiental orientada a productores y comunitarios, VE-3: Educación orientada a la prevención y mitigación de la contaminación del río Trintara, VE-4: Escolaridad.

El resultado pone en evidencia una vez más la pobre educación ambiental de los pobladores del municipio de Waspam y por la falta de capacitaciones, charlas, talleres, y programas radiales o escritos encaminados a promover la conservación del medio ambiente y la protección de fuentes de agua (nacientes, ojos de agua, áreas de recarga, entre otros).

Cuadro No. 16. Vulnerabilidad global (VG)

\begin{tabular}{|c|c|c|c|c|c|c|c|}
\hline \multirow{2}{*}{$\begin{array}{l}\text { Tipos de vulnerabilidades a } \\
\text { la contaminación }\end{array}$} & \multicolumn{6}{|c|}{ Promedio de los tipos de vulnerabilidades } & \multirow[b]{2}{*}{ Vulnerabilidad } \\
\hline & VF & VPI & VEC & VEN & VS & VE & \\
\hline Física & 1.91 & & & & & & 1.91 \\
\hline Política-Institucional & & 3.20 & & & & & 3.20 \\
\hline Ecológica & & & 2.67 & & & & 2.67 \\
\hline Económica & & & & 3.20 & & & 3.20 \\
\hline Social & & & & & 3.29 & & 3.29 \\
\hline Educativa & & & & & & 3.50 & 3.50 \\
\hline Promedio & 1.91 & 3.20 & 2.67 & 3.20 & 3.29 & 3.50 & 2.96 \\
\hline Valor Máximo definido & & & & & 4 & & \\
\hline V-Global (\%) & 39.80 & 70.00 & 58.67 & 70 & 71.2 & 75.20 & 64.15 \\
\hline $\begin{array}{l}\text { Caracterización de la } \\
\text { vulnerabilidad }\end{array}$ & $\frac{.0}{\frac{0}{0}}$ & $\frac{⿱ 乛 ⿻}{\frac{1}{2}}$ & $\frac{.00}{\frac{0}{0}}$ & $\frac{\sigma}{\frac{\sigma}{2}}$ & $\frac{⿱ 乛 ⿻}{\frac{1}{\alpha}}$ & $\frac{⿱ 乛 ⿻}{\frac{1}{2}}$ & Alta \\
\hline
\end{tabular}


Se podría decir que la educación es el indicador social más importante para el manejo de la micro cuenca, esto conlleva conocimientos sobre la utilidad de la micro cuenca para la vida social y ambiental, en todo caso aunque se implementen medidas de regulación y control estas podrían no funcionar si no se integra la participación consciente de la población.

El reto entonces está en implementar programas integrales de manejo de la micro-cuenca, con la participación comunitaria y de toda la población, y el Estado deberá garantizar alternativas de vida a la población. El objetivo final es reducir la vulnerabilidad de los recursos hídrico del micro-cuenca Trintara Tigni.

\section{Alternativas de reducción de la vulnerabilidad a la contaminación}

Las propuestas de alternativas de solución a la reducción de la vulnerabilidad a la contaminación de aguas superficiales se elaboraron a partir de los resultados del análisis de la vulnerabilidad global de la micro-cuenca del río Trintara.

Los planes y estrategias de las propuestas presentadas tienen como propósito: El desarrollo de una consciencia en la población sobre la importancia que implica para ellos la protección del área de la micro cuenca del río Trintara, a través de estrategias integrales de comunicación y educación ambiental; la promoción de la organización comunal y con base en ellos establecer iniciativas de gestión integral en coordinación con la municipalidad junto con los gobiernos territoriales y los ciudadanía en general.

\section{Conclusiones}

El clima de la micro cuenca es de tipo tropical húmedo con precipitaciones entre 1,900 y los 3,290 $\mathrm{mm}^{3}$ /año, los suelos en su mayoría con fertilidad de media a baja predominando el orden Inceptisol bien drenado, y una red de drenaje con sus tributarios bien definidos, presentando con orden de corrientes de tipo (1, 2, у 3 ), por su sistema de drenaje es una micro cuenca exorreica y de categoría multiusos.

Los suelos tienen una fertilidad media y baja, por el cual para la producción de granos básicos es entre media a baja en el área de la micro cuenca, ante ello los pobladores practican una agricultura de carácter de subsistencia y muy poco de carácter comercial.

El análisis del agua de la micro cuenca Trintara presenta un alto grado de contaminación con coliformes fecales humano y animales, por lo tanto no es apta para consumo humano. Sin embargo, en la parte alta de la micro cuenca el análisis demuestra una disminución considerable de los niveles de contaminación. 
La vulnerabilidad global del área de la micro-cuenca del río Trintara Tigni es de $2.96(64.15 \%)$, caracterizando como de alta vulnerabilidad a la contaminación. Esto se debe a que el $60 \%$ del área de la micro cuenca se encuentra poblada sin ninguna organización urbanística, una agricultura de subsistencia dispersa y la extracción libre de madera y materiales de construcción. El reto está en implementar programas integrales de manejo de la micro cuenca, con la participación comunitaria y de toda la población.

\section{Listas de referencia}

Alcaldía de Waspam, de área de recaudación de fondos (2011). Informe (documento no publicado).

Burton, Jean (2003). Integrated Water Resources Management on a Basin Level. A Training Manual. UNESCO; Ediciones MultiMondes. Canada. 240 pp.

Guerra Turrubiates Laura Elisa, (2004). La contaminación del agua, Trabajo de investigación, Preparatoria Buckingham, Universidad autónoma de México.

Ley de aguas nacionales No. 620, publicada en la Gaceta No. 169, (2007), Nicaragua.

Ley especial de delitos contra el medio ambiente y los recursos naturales ley No. 559. (2005). Nicaragua.

MINSA (2011). Informe de muestreo de agua del micro-cuenca Trintara Tigni, Departamento de sanidad e higiene.

Watler Reyes, William J. (2008). Análisis de vulnerabilidad a la contaminación del recurso hídrico en la subcuenca del río Siquirres, Costa Rica. CATIE, Turrialba, Costa Rica.

Watler Reyes, William J. (2010). Cuencas Hidrográficas: Modulo I. Maestría en Desarrollo con Identidad, con Mención en Gobernabilidad Territorial y Manejo de Bosques. uraccan, Puerto Cabezas, Volumen 110 Pag.

Wilches-Chaux, Gustavo (1989). Desastres, ecologismo y formación profesional: herramientas para la crisis. Servicio Nacional de Aprendizaje, Popayán. Disponible en: http://hum.unne.edu.ar/revistas/geoweb/Geo2/contenid7vulner7.htm\#18 